\title{
O PROTOCOLO DE MANCHESTER COMO FERRAMENTA DE MELHORA DOS SERVIÇOS DE EMERGÊNCIA
}

\section{THE MANCHESTER PROTOCOL AS A TOOL FOR IMPROVING EMERGENCY SERVICES}

\section{EL PROTOCOLO DE MANCHESTER COMO HERRAMIENTA PARA MEJORA DE LOS SERVICIOS DE EMERGENCIA}

\section{Laryssa de Farias Morais ${ }^{1}$, Carla Barbosa Arruda², Amanda Tavares Xavier ${ }^{3}$, João Victor Batista Cabral ${ }^{4}$}

Como citar esse artigo: Morais LF, Arruda CB, Xavier AT, Cabral JVB. O protocolo de Manchester como ferramenta de melhora dos serviços de emergência. Rev Enferm Atenção Saúde [Internet]. 2021 [acesso em ];10(1):e202108. doi:10.18554/reas.v10i1.4210

\section{RESUMO}

Objetivo: Descrever os benefícios do uso do Protocolo de Manchester em serviços hospitalares de emergência percebidos pelos enfermeiros classificadores. Método: Trata-se de um estudo transversal descritivo-exploratório de abordagem quantitativa, realizado com enfermeiros da unidade de emergência de um hospital de alta complexidade da cidade do Recife/PE Resultados: Verificou-se que $80 \%$ dos entrevistados perceberam benefícios para o paciente e para a melhoria da rotina do serviço, dos quais $90 \%$ verificaram redução no tempo de espera para atendimento, $70 \%$ apontaram redução na mortalidade após a implementação da classificação de risco e melhora na satisfação do usuário (40\%) e na relação profissional/paciente (20\%). Conclusão: Foi evidenciado que os profissionais conhecem e afirmam que o Protocolo de Manchester tem um grande impacto, quando se trata de benefícios e melhor mecanismo de gerenciamento, além da diminuição do risco de agravamento à saúde dos pacientes.

Descritores: Emergência; Acolhimento; Enfermagem; Classificação de Risco.

\footnotetext{
${ }^{1}$ Egressa do curso de Enfermagem do Centro Universitário da Vitória de Santo Antão, Vitoria de Santo AntãoPE. https://orcid.org/0000-0001-6356-5162, e-mail: larissamorais1937@ hotmail.com

${ }^{2}$ Egressa do curso de Enfermagem do Centro Universitário da Vitória de Santo Antão, Vitoria de Santo AntãoPE. https://orcid.org/0000-0001-9005-2784, e-mail: carlab.arruda@gmail.com

${ }^{3}$ Enfermeira. Mestre em Ciências da Saúde. Doutoranda do programa de Pós-graduação em Ciências da Saúde, Universidade de Pernambuco, Recife-PE. Docente do curso de Enfermagem do Centro Universitário da Vitória de Santo Antão, Vitória de Santo Antão, PE. https://orcid.org/0000-0001-9775-1078, e-mail: amandaxavier@hotmail.com

${ }^{4}$ Enfermeiro. Mestre em Ciências da Saúde. Doutorando do programa de Pós-graduação em Inovação Terapêutica, Universidade Federal de Pernambuco, Recife-PE. Docente do curso de Enfermagem do Centro Universitário da Vitória de Santo Antão - Vitória de Santo Antão, PE. https://orcid.org/0000-0001-8836-7875, e-mail: jvbcabral@gmail.com
} 


\begin{abstract}
Objective: To describe the benefits of using the Manchester Protocol in emergency hospital services perceived by classifying nurses. Method: This is a cross-sectional descriptiveexploratory study with a quantitative approach, carried out with nurses in the emergency unit of a highly complex hospital in the city of Recife / PE Results: It was found that $80 \%$ of the interviewees perceived benefits for the patient and for the improvement of the service routine, of which $90 \%$ found a reduction in the waiting time for care, $70 \%$ indicated a reduction in mortality after the implementation of the risk classification and improvement in user satisfaction (40\%) and in the professional / patient (20\%). Conclusion: It was evidenced that the professionals know and affirm that the Manchester Protocol has a great impact, when it comes to benefits and a better management mechanism, in addition to decreasing the risk of worsening the health of patients.
\end{abstract}

Descriptors: Emergency; Reception; Nursing; Risk rating.

\title{
RESUMEN
}

Objetivo: Describir los beneficios de utilizar el Protocolo de Manchester en los servicios hospitalarios de emergencia percibidos por la clasificación de enfermeras. Método: Este es un estudio descriptivo-exploratorio de corte transversal con un enfoque cuantitativo, realizado con enfermeras en la unidad de emergencia de un hospital altamente complejo en la ciudad de Recife / PE Resultados: Se encontró que el 80\% de los entrevistados percibieron beneficios para el paciente y para la mejora de la rutina del servicio, de los cuales el $90 \%$ encontró una reducción en el tiempo de espera para la atención, el 70\% indicó una reducción en la mortalidad después de la implementación de la clasificación de riesgos y la mejora en la satisfacción del usuario (40\%) y en el paciente (20\%). Conclusión: se evidenció que los profesionales saben y afirman que el Protocolo de Manchester tiene un gran impacto, en lo que respecta a los beneficios y un mejor mecanismo de gestión, además de disminuir el riesgo de empeorar la salud de los pacientes.

Descriptores: Emergencia; Albergar; Enfermería; Clasificación de Riesgo.

\section{INTRODUÇÃO}

A triagem com classificação de risco envolve um emaranhado processo de tomada de decisões, justificado pela criação de diversos sistemas de classificação, com o intuito de ajudar o enfermeiro na decisão classificatória da gravidade de cada caso. De forma sucinta, estes sistemas têm como objetivo diminuir o tempo de espera do usuário, através da priorização no atendimento dos casos com maior gravidade, cujo prognóstico tende a ser mais complexo devido ao atraso no atendimento. ${ }^{1}$

No ano de 2004, foi implantada a Política Nacional de Humanização (PNH) no Brasil, que tem como princípio norteador a valorização do sujeito e o fortalecimento da relação entre usuário e trabalhador. Esta política consiste em um conjunto de propostas, com finalidade de promoção da saúde populacional através de valores norteadores de autonomia e protagonismo 
dos participantes, assim como participação coletiva nas práticas de saúde. ${ }^{2}$

Em 2014, o Ministério da Saúde (MS), divulgou a Portaria 1.442, que implantou nos serviços de emergência, o Acolhimento com Classificação de Risco (ACCR). O ACCR foi implantado nos serviços de emergência, com a finalidade de excluir a lógica do atendimento por ordem de chegada. A classificação é realizada exclusivamente por enfermeiros treinados, enquanto o acolhimento é feito por qualquer profissional de saúde. No Brasil, a classificação de risco é feita com uso do Protocolo de Manchester (PM) que diferentemente de outros sistemas de classificação, visa a melhoria do vínculo entre profissional e usuário, através da escuta qualificada. ${ }^{3}$

O PM consiste em classificar os pacientes de acordo com suas necessidades de saúde mais graves. Tal classificação segue um padrão que consiste em: identificar o agravo a saúde, seguindo com pensamento crítico a respeito do grau da necessidade de atendimento e por fim, a decisão do tempo de espera do paciente, de acordo com o quadro clínico apresentado no momento da avaliação. ${ }^{4}$

A classificação de risco de acordo com o PM trabalha com um sistema de cores versus tempo, onde: Vermelho emergência, atendimento imediato; Laranja - Muito urgente, atendimento em até 10 minutos; Amarelo - urgente, paciente pode esperar até 60 minutos; Verde - Pouco urgente, podendo esperar até 120 minutos; Azul - Não urgente, podendo aguardar até 240 minutos para receber atendimento. ${ }^{5}$

Para que o ACCR seja implantado, faz-se necessário a adequação da área física e a organização do setor de emergência. Segundo o MS, o setor voltado para atendimento de emergências deve ser dividido em eixos capazes de evidenciar o nível de gravidade do paciente. Ao menos, dois eixos representantes da gravidade do pacientes devem sem implantados: vermelho (área destinada para pacientes classificados com médio e alto risco de vida) e azul (espaço voltado aos pacientes que apresentam baixo risco de vida, porém precisam de avaliação médica). ${ }^{2}$

O PM é considerado uma ferramenta que permite a identificação facilitada de pacientes críticos/graves nas unidades de urgência/emergência. $\mathrm{O}$ serviço torna-se mais organizado e capaz de atender a todos os pacientes de forma segura, sem exclusão e de acordo com a necessidade real de atendimento prioritário, fato fundamental na realidade de superlotação brasileira. ${ }^{6}$ Além disso, a operacionalização do protocolo ocorre por meio do uso de fluxogramas, o que torna o trabalho de avaliação e classificação do paciente por gravidade mais fácil. ${ }^{7}$ 
Considerando o acolhimento com classificação de risco que foi implantado em um determinado serviço de emergência com a finalidade de realizar o atendimento de acordo com as necessidades médicas mais graves, excluindo a ordem de chegada, justificou-se a necessidade de pesquisar os benefícios obtidos através do protocolo utilizado nas emergências. Espera-se que os enfermeiros constatem que a instituição obteve melhorias na qualidade do atendimento após implementação do ACCR e uso do PM, pressupondo que houve maior respaldo científico na avaliação inicial e na priorização da necessidade de intervenções, de acordo com a gravidade e risco de vida do paciente.

A identificação e análise dos benefícios que podem ser percebidos pelos enfermeiros em relação a implementação do PM pode otimizar e difundir sua aplicação nos serviços de saúde, melhorar e conscientizar o atendimento e o acolhimento da população e reduzir a cultura da resistência institucional na implantação de classificações de risco. Assim, este estudo objetivou descrever os benefícios do uso do PM em serviços hospitalares de emergência percebidos pelos enfermeiros classificadores.

\section{MÉTODO}

Trata-se de um estudo transversal descritivo-exploratório de abordagem quantitativa, realizado com os enfermeiros da unidade de emergência de um hospital de alta complexidade da cidade do Recife/PE. Foram incluídos todos os 14 enfermeiros lotados na emergência geral nos dois turnos de trabalho existentes (diurno e noturno). Desses, oito aceitaram participar da pesquisa e foram entrevistados.

Os dados foram coletados através de um instrumento do tipo check list, contendo dezesseis perguntas objetivas que caracterizavam o perfil do profissional (sexo, idade, titulação e especialidade acadêmica, tempo de trabalho, número de vínculos) e descrevia aspectos relacionados ao PM (benefícios do uso o protocolo, rotina de uso, conhecimento sobre o protocolo e política nacional de humanização).

A coleta de dados ocorreu no mês de outubro de 2018 sem agendamento prévio, todavia, com ciência da ocorrência da pesquisa, uma vez que houve anuência e comunicação pela gerência de enfermagem. $\mathrm{O}$ instrumento foi apresentado a cada participante de forma reservada e pôde ser respondido sem um tempo estabelecido para devolução. Enquanto isso, o pesquisador permaneceu na sala, aguardando a entrega do checklist devidamente respondido. $\mathrm{O}$ banco de dados foi confeccionado por meio do programa Microsoft Excel® 2010, e analisado 
segundo estatística descritiva simples (frequência e percentual).

A pesquisa ocorreu com o devido consentimento dos profissionais, através da assinatura do Termo de Consentimento Livre Esclarecido (TCLE), após autorização do serviço e aprovação do Comitê de Ética e Pesquisa sob CAAE: 02363018.6.0000.5200 e Parecer Consubstanciado $\quad \mathrm{n}^{\mathbf{0} 3}$ 3.020.206. Foi assegurado o compromisso ético da utilização dos seus dados segundo a resolução 466/12 do Conselho Nacional de Saúde (CNS).

Os resultados do estudo foram descritos em Tabelas, quanto à caracterização sociodemográfica e aptidão referida dos participantes (Tabela 1), avaliação do conhecimento sobre o Protocolo de Manchester (Tabela 2) e benefícios percebidos pelos Enfermeiros com relação ao uso do Protocolo de Manchester (Tabela 3).

\section{RESULTADOS}

A amostra do estudo foi composta por oito enfermeiros, ao qual 100\% (8) eram do sexo feminino e possuíam tempo de trabalho em serviço de emergência acima de 6 anos e $70 \%$ (5) estavam com idade acima de 50 anos. No que concerne à formação acadêmica, 20\% (2) descreveram não possuir curso de pós-graduação finalizado, enquanto $80 \%$ (6) referiram possuir pósgraduação já finalizada. Todas as pósgraduações $(100 \%)$ foram referidas como lato sensu do tipo especialização e nenhuma (0\%) do tipo strictu sensu.

Sobre o número de vínculos empregatícios, $50 \%$ (4) referiram possuir um único vínculo hospitalar e 50\% (4) relataram possuir dois vínculos hospitalares, não sendo referido por nenhum participante $(0 \%)$ um quantitativo igual ou superior a três empregos. Quanto à capacitação para utilizar o PM como acolhimento nos serviços de emergência, $100 \%$ (8) responderam que houve capacitação e treinamento sobre o manuseio do PM (Tabela 1). 
Tabela 1- Caracterização sociodemográfica e Aptidão Referida dos Participantes

\begin{tabular}{lccc}
\hline Variáveis & Categoria & N=8 & \% \\
\hline Sexo & Feminino & 8 & $100 \%$ \\
& Masculino & - & - \\
\hline Idade (em anos) & $30-40$ & - & - \\
& $40-50$ & 3 & $30 \%$ \\
\hline Tempo de Trabalho em Emergência & $>50$ & 5 & $70 \%$ \\
& De $2-4$ anos & - & - \\
& De $4-6$ anos & - & - \\
\hline Pós-Graduação & $>6$ anos & 8 & $100 \%$ \\
\hline Número de Vínculos Empregatícios & Sim & 6 & $80 \%$ \\
& Não & 2 & $20 \%$ \\
& 1 Hospital & 4 & $50 \%$ \\
\hline Capacitado para Trabalhar na & 2 Hospitais & 4 & $50 \%$ \\
Classificação de Risco? & >3 Hospitais & - & - \\
\hline
\end{tabular}

Fonte: Os autores, 2020.

Em relação ao conhecimento dos enfermeiros sobre o PM, verificou-se que $8(100 \%)$ afirmam conhecer os níveis de urgência estabelecidos pelo protocolo, subdivididos por cores a partir da gravidade, $80 \%(6)$ referem ter o conhecimento sobre a PNH do SUS (Tabela 2).

Tabela 2- Avaliação do Conhecimento sobre o Protocolo de Manchester

\begin{tabular}{llll}
\hline Variáveis & Categoria & $\mathbf{N = 8}$ & $\mathbf{\%}$ \\
\hline Conhecimentos dos Níveis de Urgência? & Sim & 8 & $100 \%$ \\
\hline Conhece a Política Nacional De Humanização (PNH)? & Não & - & - \\
\hline & Sim & 6 & $80 \%$ \\
\hline
\end{tabular}

Fonte: Os autores, 2020.

Em relação aos benefícios percebidos pelos enfermeiros com o uso do PM, verificou-se que $80 \%$ (6) dos entrevistados perceberam a ocorrência de benefícios para o paciente e em relação à melhoria da rotina do serviço emergencial. Dos respondentes, 70\%(5) asseguraram que com o uso do PM, todos os pacientes são atendidos conforme o seu risco/gravidade e que verificaram redução na incidência de mortalidade no serviço após a implementação da classificação de risco. Verificou-se que ainda há uma dificuldade na compreensão dos pacientes em relação ao tempo de espera, pois $80 \%(6)$ dos enfermeiros afirmam perceber que os pacientes não compreendem os critérios de classificação e 
sua relação com o tempo que deve ser esperado para o atendimento (Tabela 3).

De acordo com os benefícios que foram percebidos em relação ao paciente, $3(40 \%)$ concordam que houve diminuição de sequelas, $40 \%$ (3) apontam a melhora na satisfação com o atendimento e 20\%(2) acreditam que houve benefícios na relação profissional/paciente. Em relação ao serviço emergencial, 90\%(7) afirmam que houve benefício no quesito tempo de espera, que foi diminuído (Tabela 3).

Tabela 3- Benefícios Percebidos pelos Enfermeiros com uso do Protocolo de Manchester

\begin{tabular}{|c|c|c|c|}
\hline Variáveis & Categoria & $\mathbf{N}=\mathbf{8}$ & $\%$ \\
\hline \multirow[t]{3}{*}{ Benefícios aos Pacientes? } & Sim & 6 & $80 \%$ \\
\hline & Não & 2 & $20 \%$ \\
\hline & Não Sei & - & - \\
\hline \multirow[t]{3}{*}{ Benefícios à Rotina Emergencial? } & Sim & 6 & $80 \%$ \\
\hline & Não & 2 & $20 \%$ \\
\hline & Não Sei & - & - \\
\hline Assegura que todos sejam Atendidos & Sim & 5 & $70 \%$ \\
\hline \multirow[t]{2}{*}{ Conforme o Risco? } & Não & 3 & $30 \%$ \\
\hline & Não Sei & - & - \\
\hline \multirow{3}{*}{$\begin{array}{l}\text { Houve redução da Incidência de Mortalidade } \\
\text { após Implementação da Classificação de } \\
\text { Risco? }\end{array}$} & Sim & 5 & $70 \%$ \\
\hline & Não & 2 & $20 \%$ \\
\hline & Não Sei & 1 & $10 \%$ \\
\hline Há dificuldade dos Pacientes na & Sim & 6 & $80 \%$ \\
\hline \multirow[t]{2}{*}{ Compreensão do Tempo/espera? } & Não & 2 & $20 \%$ \\
\hline & Não Sei & - & - \\
\hline \multirow{3}{*}{$\begin{array}{l}\text { Benefícios Percebidos em Relação ao } \\
\text { Paciente }\end{array}$} & Sequelas diminuídas & 3 & $40 \%$ \\
\hline & $\begin{array}{l}\text { Satisfação no } \\
\text { atendimento }\end{array}$ & 3 & $40 \%$ \\
\hline & $\begin{array}{l}\text { Melhoria na relação } \\
\text { profissional/paciente }\end{array}$ & 2 & $20 \%$ \\
\hline \multirow{3}{*}{$\begin{array}{l}\text { Benefícios Percebidos em Relação ao } \\
\text { Serviço Emergencial }\end{array}$} & Menos tempo de espera & 7 & $90 \%$ \\
\hline & Demanda diminuída & - & - \\
\hline & Melhoria na qualificação & 1 & $10 \%$ \\
\hline
\end{tabular}

Fonte: Os autores, 2020.

\section{DISCUSSÃO}

Verificou-se que a totalidade dos respondentes era do sexo feminino, com idade acima de 50 anos e com mais de 6 anos de experiência/trabalho em serviços de emergência. Tais achados são corroborados por outros estudos, que destacam a enfermagem como uma profissão de predomínio feminino ${ }^{1-3}$ e com idade superior aos $50 \operatorname{anos}^{8}$ e com expressivo tempo de serviço no exercício da profissão. ${ }^{9}$ É importante destacar que com o avanço no número e acesso ao ensino superior no Brasil, os cursos de saúde apresentam uma 
significativa procura, sendo o curso de enfermagem um dos destaques nesse cenário, o que aponta para uma transição etária na profissão.

No que concerne à formação, a maioria referiu possuir pós-graduação lato sensu. O resultado desta variável não corrobora com o achado de outro estudo ${ }^{10}$, uma vez que apenas $19,7 \%$ dos participantes concluíram algum curso de pós-graduação. A busca por aprimoramento e qualificação após a formação da graduação ainda é heterogênea no país, todavia, nota-se uma crescente procura por cursos de especialização, ofertados em modalidades presenciais ou a distância. Além disto, destaca-se o importante papel do SUS na educação permanente dos seus colaboradores através de parcerias com instituições e organizações educacionais para $\mathrm{o}$ fomento e qualificação dos profissionais que estão na ponta.

Em se tratando do número de vínculos empregatícios, metade possuem de um a dois vínculos. Em estudo feito no estado do Rio Grande do Norte, também na região nordeste $^{7}$ viu-se que em sua maioria, os entrevistados referem possuir o mesmo número de vínculos que o estudo em pauta. Tal fato apresenta a realidade da maioria dos profissionais da enfermagem do Brasil, pois reflete a necessidade por mais um vínculo empregatício em virtude dos baixos salários e de condições de trabalho, muitas vezes, desfavoráveis.

Sobre a capacitação para trabalhar na classificação de risco, todos os entrevistados referiam estar/serem capacitados. Verifica-se que o enfermeiro tem sido responsável pelo procedimento de ACCR sustentado por protocolos. Então, de acordo com a cartilha do PNH um dos prérequisitos necessários para a implantação da $\mathrm{CR}$, é a qualificação da equipe de profissionais que vão fazer parte do acolhimento, mediante treinamento específico e utilização de protocolos preestabelecidos. ${ }^{11}$ Destaca-se que para se tornar classificador, o enfermeiro deve ser treinado e acompanhado periodicamente de acordo com a ferramenta de classificação, pois assim este obterá habilitação para o manejo do instrumento e também para aplicação de raciocínio clínico, tornando o processo menos mecanizado e mais humanizado. $^{12}$

Verificou-se que a totalidade da amostra tem conhecimento correto sobre os níveis de urgência descritos pelo PM a partir da gravidade e sinais e sintomas de cada paciente. A diretriz de ACCR impõe que o usuário seja acolhido no serviço hospitalar e atendido de acordo com os critérios de riscos pré-estabelecidos, os quais, através da consulta de enfermagem, permitem ao profissional chegar a uma classificação do potencial de risco de cada caso, de acordo 
com o sistema de cores ${ }^{3}$. Tal entendimento é crucial para o cotidiano do trabalho, uma vez que permite ao profissional dinamizar a sua prática e determinar um fluxo seguro de atendimento.

Observou-se que a maioria reconhece a PNH do MS e que sua aplicação permite pôr em prática os princípios do SUS, no cotidiano dos serviços de saúde. A PNH resultou em mudanças significativas nos serviços emergenciais, nos quais as formas de condutas foram alteradas. A construção de políticas públicas de saúde nas últimas décadas enfatiza a necessidade de implantar boas práticas de saúde voltadas para a integralidade do cuidado. Nessa perspectiva, o serviço de ACCR contribui para o adequado atendimento nas unidades de saúde. ${ }^{4}$

Para alguns autores, ainda há dificuldade quando se trata do conhecimento do enfermeiro na classificação de risco em relação a PNH, a qual propõe que o ACCR deve ser dado a todos os usuários, mesmo os que estão em casos não graves e que podem ter seus problemas resolvidos em uma unidade básica de saúde. O conhecimento e integração da classificação com parte integrante da $\mathrm{PNH}$ é fundamental, pois apenas o uso do PM sem a PNH, torna-se insuficiente, pois juntos envolvem sinais, sintomas e aspectos culturais e sociais, que podem ser um ponto fundamental para a adequada avaliação de risco de cada pessoa de forma diferenciada. ${ }^{6,13}$

Pôde-se comprovar que após implementação do PM, foram percebidos benefícios pelos enfermeiros, tanto em relação aos aspectos dos pacientes, como em relação à rotina da instituição. A implantação da classificação de risco traz contribuições positivas no âmbito de cuidado à saúde, ${ }^{14}$ facilitando o atendimento, sendo uma importante ferramenta na priorização dos casos graves, proporcionando segurança para a enfermagem e para os pacientes, uma vez que já foram avaliados previamente enquanto estão aguardando o atendimento médico. ${ }^{11,6}$

Pela percepção dos enfermeiros, o PM assegura que todos os pacientes sejam atendidos conforme o seu real risco. A partir do ACCR, os profissionais têm melhor percepção dos pacientes que apresentem maior risco, podendo ser otimizados com a utilização do protocolo, tornando este importante e necessário instrumento na prática diante dos agravos clínicos. ${ }^{10}$

Outro ponto de benefício apontado pelos enfermeiros, foi a diminuição na incidência de mortalidade após implementação do protocolo, uma vez que, de acordo com a política de classificação de risco, o profissional deve reavaliar $\mathrm{o}$ paciente, para verificar se houve melhora ou piora do caso e se necessário, mudar o 
sistema de cores inicial e reclassificar o paciente caso este apresente piora do estado clínico. Em outro estudo ${ }^{15}$ após a implantação do PM, pode-se observar que houve diminuição na mortalidade dos pacientes que foram classificados, contribuindo para redução das mortes por falta de atendimento.

Ressalta-se que ainda há uma dificuldade de compreensão dos pacientes no que diz respeito ao tempo de espera para $\mathrm{o}$ atendimento, todavia em estudo em Minas Gerais $^{16}, 87 \%$ dos usuários pesquisados se mostraram satisfeitos com o serviço, no que diz respeito ao tempo de espera para obter o atendimento. Destaca-se que a maioria dos ocupantes da emergência poderiam ter seu problema resolvido na atenção básica, contribuindo assim na diminuição das longas filas de espera para os que realmente necessitam do serviço emergencial.

Pela percepção dos respondentes deste estudo, em relação aos pacientes, verificou-se que houve diminuição nas sequelas, satisfação no atendimento e melhoria na relação profissional/paciente. Segundo estudo realizado por Souza et al., $(2014)^{17}$ após a implantação do PM os usuários foram diferenciados de acordo com seu nível de urgência, diminuindo assim a grande demanda nos serviços e os que foram classificados com a cor azul, foram instruídos a procurar a atenção básica para solução dos problemas, diminuindo assim o tempo de espera por atendimentos dos casos não graves.

Para os enfermeiros de um prontoatendimento da região sul do Brasil, o atendimento com priorização aos pacientes com possíveis risco de agravos é visto como uma das contribuições positivas do PM, assegurando o atendimento às urgências e diminuindo as sequelas e os riscos de agravamento decorrentes do tempo de espera prolongado além de contribuir para que o usuário se sinta seguro ${ }^{11}$ pois é realizada uma escuta qualificada e assim uma maior aproximação entre o usuário e a equipe de saúde, garantindo que ele não deixe o serviço sem receber algum tipo de atendimento.

Destaca-se que para que haja a construção de acolhimento entre os usuários e trabalhadores e uma qualidade nessa relação, faz-se necessário a implantação de sistemas/programas de classificação de risco, podendo assim atingir melhores patamares na qualidade do atendimento. $\mathrm{O}$ PM é um exemplo de instrumento que permite o alcance de bons resultados e vem sendo utilizado em diversos países do mundo, com diferentes realidades. Sua confiabilidade é considerada aceitável e há concordância quanto aos benefícios resultantes de sua implementação. ${ }^{18-20}$

\section{CONCLUSÕES}


Conhecer a percepção e as expectativas dos profissionais que fazem o atendimento no serviço de ACCR é um elemento importante para avaliação dos serviços emergenciais. No presente estudo foi evidenciado que os profissionais conhecem e afirmam que o PM tem um grande impacto, quando se trata de benefícios e melhor mecanismo de gerenciamento, além da diminuição do risco de agravamento à saúde dos pacientes.

Nota-se que os profissionais de enfermagem possuem uma preocupação relacionada a qualidade do ACCR nos serviços de emergência, assim, torna-se importante a divulgação de estudos sobre o tema, para que possam contribuir para um melhor planejamento e eficácia deste sistema. Além disso, instituições que ainda não implantaram a classificação de risco, podem se basear nas experiências compartilhadas do espaço científico, adaptando-as as suas reais necessidades.

Destacam-se como limitações o tamanho amostral e o fato de o estudo ter sido realizado em apenas um hospital, que apesar de ser referência para emergências clínicas e traumáticas, pode não refletir com exatidão as características de outros serviços de saúde.

\section{REFERÊNCIAS}

1. Hinson JS, Martinez DA, Scmitz PSK, Toerper M, Radu D, Scheulen J, et al. Accuracy of emergency department triage using the Emergency Severity Index and independent predictors of under-triage and over-triage in Brazil: a retrospective cohort analysis. Int J Emerg Med. [Internet]. 2018 [citado em 07 out 2020]; 11(3):1-10. Disponível em: https://intjem.springeropen.com/articles/10 .1186/s12245-017-0161-8

2. Prudêncio CPG, Monteiro RAN, Ribeiro BCM, Gomes MSM, Manhães LSP. Percepção de enfermeira(o)s sobre acolhimento com classificação de risco no serviço de pronto atendimento. Rev Baiana Enferm. [Internet]. 2016 [citado em 13 ago 2019]; 30(2):1-10. Disponível em: https://portalseer.ufba.br/index.php/enferm agem/article/view/14917/pdf_46

3. Costa MAR, Versa GLGS, Bellucci JA, Inoue KC, Sales CA, Matsuda LM. Acolhimento com classificação de risco: avaliação de serviços hospitalares de emergência. Esc Anna Nery Rev Enferm. [Internet]. 2015; [citado em 30 ago 2019]; 23(1):491-7. Disponível em: http://www.scielo.br/pdf/ean/v19n3/14148145-ean-19-03-0491.pdf

4. Ebrahimi M, Mirhaghi A, Mazlom R, Heydari A, Nassehi A, Jafari M. The role descriptions of triage nurse in emergency department: a Delphi study. Scientifica (Cairo) [Internet]. 2016 [citado em 15 jul 2020]; 2016(ID5269815):1-6. doi:10.1155/2016/5269815

5. Mackway-Jones K, Marsden J, Windle J. Emergency triage: Manchester triage group. 3rd ed. Cowley, Oxford: BMJ Books; 2014.

6. American Academy of Pediatrics Committee on Pediatric Emergency Medicine. Overcrowding crisis in our nation's emergency departments: is our safety net unraveling? Pediatrics [Internet]. 2004 [citado em 15 jul 2020]; 114(3):87888. doi:10.1542/peds.2004-1287

7. Roncalli AA, Oliveira DN, Silva ICM, Brito RF, Viegas SMF. Protocolo de Manchester e população usuária na classificação de risco: visão do enfermeiro. Rev Baiana Enferm. [Internet]. 2017. 
[citado em 20 set 2019]; 31(2):e16949.

Disponível em:

https://portalseer.ufba.br/index.php/enferm agem/article/view/16949/14511. doi: 10.18471/rbe.v31i2.16949

8. Freitas RJM, Pereira MFA, Lima CHP, Melo JN, Oliveira KKD. A violência contra os profissionais da enfermagem no setor de acolhimento com classificação de risco. Rev Gaúch Enferm. [Internet]. 2017 [citado 30 set 2019]; 38(3):e62119.

Disponível em:

http://www.scielo.br/pdf/rgenf/v38n3/0102 -6933-rgenf-38-3-e62119.pdf

9. Soares ACL, Brasileiro M, Souza DG. Acolhimento com classificação de risco: atuação do enfermeiro na urgência e emergência. Rev Recien [Internet]. 2019 [citado em 07 out 2020]; 8(22):22-33. Disponível em:

https://recien.com.br/index.php/Recien/arti cle/view/245/pdf

10. Oliveira JLC, Gatti AP, Barreto MS, Bellucci Junior JA. Acolhimento com classificação de risco: percepções de usuários de uma unidade de pronto atendimento. Texto \& Contexto Enferm. [Internet]. 2017 [citado 03 dez 2019]; 26(1):e0960014. Disponível em: http://www.scielo.br/pdf/tce/v26n1/pt_010 4-0707-tce-26-01-0960014.pdf

11. Guedes HM, Martins JCA, Chianca TCM. Valor de predição do Sistema de Triagem de Manchester: avaliação dos desfechos clínicos de pacientes. Rev Bras Enferm. [Internet]. 2015 [citado em $03 \mathrm{dez}$ 2019]; 68(1):45-51. doi:10.1590/00347167.2015680107p

12. Bellucci-Júnior JA, Vituri DW, Versa GLGS, Furuya PS, Vidor RC, Matsuda LM. Acolhimento com classificação de risco em serviço hospitalar de emergência: avaliação do processo de atendimento. Rev Enferm UERJ. [Internet]. 2015. [citado em 13 ago 2019]; 23(1):82-7. doi:10.12957/reuerj.2015.4976 13. Versa GLGS, Vituri DW, Buriola AP, Oliveira CA, Matsuda LM. Avaliação do acolhimento com classificação de risco em serviço de emergência hospitalar. Rev
Gaúch Enferm. [Internet]. 2014. [citado em 13 ago 2019]; 35(3):21-28. Disponível em: http://www.scielo.br/scielo.php?pid=S198 3-

14472014000300021\&script=sci_arttext\&t $\operatorname{lng}=$ pt. doi:10.1590/1983-

1447.2014.03.45475

14. Raich M, Lorenzoni N, Angerer S, Nöhammer E, Stummer H. Nurses' experiences with the implementation of a Manchester Triage System in an emergency department. Eur J Public Health [Internet]. 2019 [citado em 07 out 2020]; 29(Suppl 4):ckz186.425. Disponível em: https://doi.org/10.1093/eurpub/ckz186. 425

15. Gonçales PC, Pinto-Júnior D, Salgado PO, Chianca TCM. Relationship between risk stratification, mortality and length of stay in a emergency hospital. Invest Educ Enferm. [Internet]. 2015. [citado em 28 jun 2020]; 33(3):424-31. Disponível em: https://www.ncbi.nlm.nih.gov/pubmed/285 69949

16. Silva PL, Paiva L, Faria VB, Ohl RIB, Chavaglia SRR. Acolhimento com classificação de risco do serviço de prontosocorro adulto: satisfação do usuário, São Paulo, SP. Rev Esc Enferm USP. [Internet]. 2016 [citado em 22 jun 2020]; 50(3):427-33. Disponível em: http://www.scielo.br/pdf/reeusp/v50n3/pt 0080-6234-reeusp-50-03-0427.pdf 17. Souza TH, Andrade SR. Acolhimento com classificação de risco: um indicador da demanda emergencial de um serviço hospitalar. Cogitare Enferm. [Internet]. 2014 [citado em 20 set 2019]; 19(4):701-8. Disponível em:

https://revistas.ufpr.br/cogitare/article/view 135941

18. Mirhaghi A, Mazlom R, Heydari A, Ebrahimi M. The reliability of the Manchester Triage System (MTS): a metaanalysis. J Evid Based Med. [Internet]. 2017 [citado em 15 jul 2020]; 10(2):129135. doi:10.1111/jebm. 12231 19. Azeredo TRM, Guedes HM, Almeida RAR, Chianca TCM, Martins JCA. Efficacy of the Manchester Triage System: 
a systematic review. Int Emerg Nurs.

[Internet]. 2015 [citado em 29 jun 2020];

23(2):47-52. doi:

10.1016/j.ienj.2014.06.001

20. Zachariasse JM, Seiger N, Rood PPM, Alves CF, Freitas Paulo, Smit FJ, et al.

Validity of the Manchester Triage System in emergency care: a prospective

observational study. PLos ONE [Internet].

2017 [citado em 07 out 2020]; 12(2):

e0170811. Disponível em:

https://www.ncbi.nlm.nih.gov/pmc/articles

/PMC5289484/pdf/pone.0170811.pdf

RECEBIDO: $18 / 12 / 2019$

APROVADO: 27/01/2021

PUBLICADO: 06/2021 